\title{
Myosin cleft movement and its coupling to actomyosin dissociation
}

Paul B. Conibear ${ }^{1}$, Clive R. Bagshaw ${ }^{1}$, Piotr G. Fajer ${ }^{2}$, Mihály Kovács ${ }^{3,4}$ and András Málnási-Csizmadia ${ }^{1,4}$

${ }^{1}$ Department of Biochemistry, University of Leicester, Leicester LE1 7RH, UK.

${ }^{2}$ Institute of Molecular Biophysics, Florida State University, Tallahassee FL32310, USA,

${ }^{3}$ Present address; Laboratory of Molecular Cardiology, NHLBI, Bethesda, MD 20892 1762, USA.

${ }^{4}$ Department of Biochemistry, Eötvös University, H-1117 Budapest, Hungary.

It has long been known that binding of actin and nucleotides to myosin are antagonistic, an observation that led to the biochemical basis for the crossbridge cycle of muscle contraction. Thus ATP binding to actomyosin causes actin dissociation, while actin binding to the myosin accelerates ADP and phosphate release. Structural studies have indicated that communication between the actin and nucleotide binding sites involves the opening and closing of the cleft between the upper and lower $50 \mathrm{~K}$ domains of the myosin he ad. Here we test the proposal that the cleft responds to actin and nucleotide binding in a reciprocal manner and show that cleft movement is coupled to actin binding and dissociation. We monitored cleft movement using pyrene excimer fluorescence from probes engineered across the cleft.

In recent years the structural basis of the actomyosin crossbridge cycle ${ }^{1}$ has been defined in increasing detail by X-ray crystallography and electron microscopy ${ }^{2-9}$. 
However, while crystal structures of myosin motor domains have been obtained for a number of nucleotide-bound states, the nature of the actin binding interaction has not been observed directly to date $2,3,8,10,11$. Residues involved in actin binding were identified by fitting the envelope of the myosin crystal structure into the electron micrograph density of decorated actin filaments. Initial docking attempts suggested that the cleft between the upper and lower 50K myosin domains might close on forming the rigor state ${ }^{12}$. This approach has been refined to the point of identifying a potential hinge point around switch 1 at the myosin nucleotide site that allows the upper 50K domain to rotate towards the lower $50 \mathrm{~K}$ domain ${ }^{7}$. Recent crystallographic studies support this concept ${ }^{6,8,9}$. While structural evidence is mounting in favour of the cleft closure model, it is important to test this idea in solution. Structural data alone provides only a static picture and the proposed transitions between states have been made by inference. We have introduced cysteine residues at position 416 and 537 across the cleft (Dictyostelium discoideum, Dd, myosin II sequence) in a cysteine-deficient ${ }^{13}$ myosin background (Fig 1a,b). Labelling these cysteine residues with N-1-pyrene iodoacetamide resulted in a species that showed extensive excimer (excited state dimer) fluorescence. Excimer flourescence was sensitive to actin and ATP binding and allowed relative movement of the residues at 416 and 537, and hence the cleft, to be monitored in kinetic assays. Previous studies of a tryptophan residue at position 425 in smooth muscle myosin (equivalent to 416 in Dd sequence), indicated that ATP induced a cleft opening ${ }^{14}$, however the fluorescence changes induced in the presence of actin are difficult to interpret owing to the contribution from actin tryptophan residues ${ }^{15}$.

\section{Results}

\section{Modelling the cleft}

The rigor structure, as modelled by Holmes et al. ${ }^{7}$, indicates that the $\alpha$-carbon distance between residues 416 and 537 (Dd numbering) is $11 \AA$, compared to 17 to $19 \AA$ in the apo and nucleotide-bound states of myosin in the absence of actin. Modelling shows that pyrene residues attached via acetamido-cysteine linkers can span these distances to form dimers without steric clashes with protein residues (Fig. 1c,d). Excimers arise 
from a precise planar arrangement of one pyrene ring in an excited state with another in the ground state, staggered by $1.3 \AA$ and separated by $3.5 \AA{ }^{16,17}$. Dimerization of the pyrene rings in other orientations leads to fluorescence quenching ${ }^{17}$. Excimer formation can arise from six different ring combinations, with respect to the relative location of the $\mathrm{C} 1$ atom in each pyrene ring (i.e. dependent on whether the $\mathrm{C} 1$ atoms are cis or trans to each other, the pyrene rings lie parallel or antiparallel and the stagger of the rings in the antiparallel case). These combinations lead to different span ranges, with the extremes of the parallel-cis-excimer satisfying an $\alpha$-carbon separation of 0 to $12 \AA$ while the trans-antiparallel-excimer can accommodate a span of 10 to $23 \AA$. Model building and molecular dynamic simulations confirm that the open state (M.ADP.BeF $F_{x}$ ) favours dimers with antiparallel rings, while the modelled rigor state, with a closed cleft $^{7}$, favours parallel rings (Fig. 1c,d). Molecular dynamics calculations of the potential of these states to form excimers is not straightforward. Some ground-state dimers may reorient during the excited state to form excimers while othe rs will lead to quenched fluorescent state. Whether the rigor- to apo-state transition is accompanied by a net increase or decrease in excimer formation therefore cannot be easily predicted. Nevertheless structural modelling (Fig. 1) shows that the excimers which may exist in the cleft-open and cleft-closed structures involve different ring arrangements and therefore it is likely there will be a change the extent of excimer formation if the cleft closes.

\section{Pyrene excimer formation}

Labelling the S416C/N537C Dd myosin motor domain mutant with N-(1-pyrene) iodoacetamide gave a fluorescent derivative (pyrene-motor domain; pyr-Md) in which the emission occurred as a broad peak at $486 \mathrm{~nm}$ corresponding to a pyrene excimer (Fig. 2). This result could only arise if both cysteines residues were labelled and were in close proximity. The excimer peak represented up to $96 \%$ of the intensity (excitation at $365 \mathrm{~nm}$ ) at $\mathrm{pH}$ 7.5. Excitation at lower wavelengths caused an increase in the monomer peaks and reduction in the excimer peak. Excitation wavelength-dependent excimer emission is indicative of ground-state pyrene dimers ${ }^{17}$. Time-resolved measurements showed that excimers were formed within $<2$ ns of the excitation pulse (Fig 3a), 
suggesting the pyrene rings were pre-dimerized or in close proximity, in the ground state $^{17}$. Time-resolved spectra were constructed by repeated pulses over an emission wavelength range of 370 to $520 \mathrm{~nm}$ (Fig 3b) and show that the excimer state is not in reversible equilibrium with the monomer state during its lifetime.

Addition of F-actin to the pyr-Md gave a $60 \%$ quench in the excimer fluorescence (Fig. 2a). The monomer peaks showed a small increase, but overall there was a large $(>50 \%)$ loss in total pyrene emission intensity. This contrasts with the effect of denaturation of pyr-Md, which was accompanied by a greater gain in monomer emission (Fig. 2b). Time-resolved measurements showed that actin addition did not change the excimer lifetime (40 ns), but reduced the amplitude of the transient (Fig 3a). Hence the intensity loss in the steady-state spectrum (Fig. 2a) is not a result of collisional quenching of the excimer state, but rather a reduced extent of excimer formation. This result indicates that actin reduces the ability of the ground-state pyrene dimer to reorient to give an excimer state, presumably through changes in the relative separation of residues 416 and 537 i.e. the degree of cleft closure. Titration of the excimer emission signal with [actin] yielded a $\mathrm{K}_{\mathrm{d}}$ of $0.4 \mu \mathrm{M}$ for actin, but some excimer still remained at saturating [actin]. Stopped-flow profiles on mixing actin and pyr-Md showed that the loss of excimer fluorescence and rise in light scatter signal were mirror images (Fig. 4a). Thus the excimer signal monitors actin binding to yield a rate constant of $0.34 \mu \mathrm{M}^{-1} . \mathrm{s}^{-1}$, while the dissociation rate constant, determined by displacement of pyr-Md with excess unlabelled Md was $=0.14 \mathrm{~s}^{-1}$. These values indicate a 4-fold weaker actin binding than for the native Dd motor domain ${ }^{18}$.

Addition of ATP to the acto-pyr-Md construct reversed the actin-induced loss in excimer emission intensity (Fig 2a). In order to interpret the changes induced by ATP, interaction of the pyr-Md with nucleotides in the absence of actin was also characterized. Here, ATP had a relatively small effect on the emission spectrum of pyrMd (Fig. 2b). In stopped-flow experiments at pH 7.5, the excimer peak was enhanced by $10 \%$ on mixing with ATP but the signal partially recovered $\left(0.2 \mathrm{~s}^{-1}\right)$ to give about an $8 \%$ enhancement in the steady state. The enhancement phase was biphasic, with approximately equal amplitude contributions and both rate constants increased linearly with [ATP]. At $500 \mu \mathrm{M}$ [ATP] the rate constants were $350 \mathrm{~s}^{-1}$ and $26 \mathrm{~s}^{-1}$ (Fig 4b). Although the latter is close to the effective hydrolysis rate constant, the slow phase 
proved to be a distinct process. The slowly hydrolysable analog ATP $\gamma \mathrm{S}{ }^{19}$ gave similar rate constants (within factor of 2) and amplitudes (within 2\%) to those with ATP for excimer enhancement across the same concentration range, therefore both phases represent binding steps ${ }^{20}$. It is notable that the excimer signal change is in the opposite direction to that induced by actin binding, suggesting the cleft movement may also be in an opposite direction. The pyr-Md construct contained the full complement of native tryptophan residues (cf. our previous studies with a single tryptophan constructs ${ }^{19,20}$ ). ATP induced 5\% enhancement in tryptophan fluorescence of pyr-Md, with a rate constant of $10 \mathrm{~s}^{-1}$, that we assign to the effective hydrolysis step ${ }^{19}$. These data indicate that the hydrolysis step, coupled with switch 2 open-closed transition, is only slowed down by 2 to 3 fold for pyr-Md compared with the wild-type motor domain.

In the presence of actin, the amplitude of the excimer fluorescence change on mixing with ATP was about 3 fold larger than in its absence (Fig. 4b), but the rate constants were similar (283 and $33 \mathrm{~s}^{-1}$ at $500 \mu \mathrm{M}$ ATP). The dissociation rate was monitored by light scattering which revealed at least a biphasic profile with rate constants of $100 \mathrm{~s}^{-1}$ and $2 \mathrm{~s}^{-1}$, as found previously for wild-type Dd motor domain ${ }^{18}$. However, a detailed comparison was difficult due to a flow artefact in the first few milliseconds and a variable contribution of the slow phase in light scatter signal that we previously assigned to reversal of filament bundling of the rigor complex ${ }^{18}$. Using other nucleotides, such as AMP.PNP, ATP $\gamma \mathrm{S}$ and ADP, provided a better test of the correlation between the pyrene excimer fluorescence and light scattering. These analogs result in slower actin dissociation ${ }^{21,22}$ and gave a correspondingly slower enhancement in excimer fluorescence. In Fig 4c, the excimer and inverted light scatter profiles were normalized to the observed amplitude after 10 seconds. The excimer fluorescence appears to lead the light scatter, but this property was dependent on the normalisation procedure and the variable contribution of slow phase in the light scatter. In some preparations the light scatter profile induced by ATP practically paralleled the fast phase of the excimer signal, whereas the slow phase of the eximer signal occurred after dissociation. In no preparations did the light scatter change exceed the fast phase of the excimer signal. A more robust property was the relative effect of different nucleotides on both excimer and light scatter kinetics, which differed by two orders of magnitude (Fig 4c). The cleft movement monitored by the pyrene label is therefore kinetically 
competent for it to be a necessary step for actin dissociation. In the absence of actin, ADP induced a small enhancement in excimer fluorescence of pyr-Md, about half the amplitude seen with ATP (i.e. 4\% overall). In the case of ATP $\gamma \mathrm{S}$ and ADP, the excimer enhancement was significantly slower in the presence of actin than in its absence.

A slow enhancement in excimer fluorescence of the acto-Md complex on mixing with ADP was still observed in nucleotide preparations that were pre-treated with glucose and hexokinase, although it is difficult to eliminate contaminant ATP entirely. Previous studies revealed a thermodynamic coupling ratio (i.e. the ratio of the actomyosin dissociation constants in the presence and absence of ADP) of about 20 for Dd myosin II, and thus ADP is expected induce limited actin dissociation at $\mu \mathrm{M}$ protein concentrations $^{18,23}$. The weaker binding of actin to the M.ADP complex is largely due to an increase in the actin dissociation rate constant from A.M.ADP compared with A.M, with less than a two-fold effect on the association rate constant of actin. This property contrasts with case of vertebrate skeletal myosin where the thermodynamic coupling ratio is larger ${ }^{23}$ and ADP slows down the association rate by an order of magnitude ${ }^{22,24}$.

\section{Discussion}

Site-specific labelling via introduced cysteine residues in a low cysteine background provides a powerful means of probing protein structure-function relationships. The cysteine-deficient construct, used as a start point in our studies, rescued the phenotype when expressed as the complete myosin heavy chain in Dd myosin II-null cells ${ }^{13}$. While cysteine-mutations and pyrene-labelling perturb some rate constants within the actomyosin cycle by 2 to 5 fold, the basic properties of the Lymn-Taylor cycle ${ }^{1}$ remain. It appears that the apo-state cleft is near fully open (or a distribution of states exist biased towards the open state) and that nucleotide binding promotes a small shift towards the fully-open state (high excimer fluorescence), whereas actin binding favors a more substantial movement to give a state in which pyrene dimers with quenched fluorescence predominate (presumed closed cleft). Structural studies suggest that 
movement of switch 1 towards its closed state (where it contacts the nucleotide through the $\mathrm{Mg}^{2+}$ and $\gamma$-phosphate, if present) is accompanied by opening of the myosin cleft and weakening of the actin binding site $\mathrm{e}^{7,8}$. Thus Mg.ADP provides less of a driving force towards the cleft-open state and hence gives an overall more limited and slower dissociation of actin compared with Mg.ATP, which strongly favours cleft opening. Here we show that these concepts apply in solution by direct observation of the cleft conformation. In the case of the Dd myosin II motor, the apo cleft state appears near fully open and ADP binding induces a marginal further opening and hence little reduction in the pyr-Md association rate with actin. In the absence of actin, nucleotide binding to pyr-Md causes a rapid shift to the switch 1 fully-closed sate, whereas in the presence of actin, the cleft is clamped shut so that nucleotides that interact more weakly with switch 1 (ATP $\gamma \mathrm{S}$ and ADP) result in a reduced rate in cleft opening. This approach should enable the cleft conformation in other myosins to be monitored and thus test, for example, the idea that the apo state of myosin $\mathrm{V}$ favors the cleft-closed state ${ }^{9}$.

\section{Methods}

Plasmid construction. Cysteine residues were introduced into a cysteine-light construct $^{13}$, provided by Dr W. M. Shih and Prof. J. A. Spudich. This cysteine-light Dd myosin-II S1 gene fragment, contained the mutations (C49S, C312Y, C442S, C470I, C599L, and C678Y) in the pTIKL-Myo vector, leaving the native C655 as a buried nonreactive cysteine residue. The motor domain coding fragment was amplified by PCR. After KpnI and BpiI digestion, the fragment was subcloned into the pDXA-M761 vector replacing its KpnI-BpiI motor domain coding fragment ${ }^{25}$. The $\mathrm{N}$-terminal sequence of Md was modified to MDGTP where P corresponds to P3 of the native sequence. The Cterminal sequence was the same as in the M761 construct and contained a His 8 fusion for ease of purification ${ }^{26,27}$. After cloning, the PCR amplified fragment was sequenced. S416C and N537C mutations were introduced by the megaprimer method ${ }^{28}$. 
Dictyostelium Culture and Protein Preparation. Dd AX2 ORF+cells were cultured and electroporated as described ${ }^{19}$. The S416/N537 myosin construct was purified on a Ni column and stored in $10 \mathrm{mM}$ DTT. $5 \mu \mathrm{M}$ protein was dialysed 3 times against argon purged buffer comprising $40 \mathrm{mM} \mathrm{NaCl}, 20 \mathrm{mM}$ Hepes, $2 \mathrm{mM} \mathrm{MgCh}, \mathrm{pH} 7.5$ for 4 hours at $4{ }^{\circ} \mathrm{C}$. The protein was then mixed with a 15 -fold excess of $\mathrm{N}$-1-pyrene iodoacetamide (Molecular Probes) in DMSO and incubated in the dark and on ice for 12 hours. The sample was centrifuged to remove insoluble pyrene and treated with $10 \mathrm{mM}$ DTT at room temperature for 1 hour. Free pyrene was removed using a G-50 Sephadex spin-column ( Nick $^{\mathrm{TM}}$ column, Amersham Biosciences) or by FPLC using a Superdex gel-filtration column (Pharmacia). Based on the pyrene absorption spectrum and protein concentration, labelling was of the order of $1 \mathrm{~mol}$ pyrene/mol motor domain. However the small pyrene monomer fluorescence signal suggested there was little singly labelled pyr-Md present or the fluorescence from this species was highly quenched. It is possible that labelling is cooperative due to pyrene ring interaction or pyrene incorporation is limited by prior disulphide formation between the introduced cysteine residues. The basal and actin-activated steady-state ATPase rates of pyr-Md were $0.035 \mathrm{~s}^{-1}$ and $0.5 \mathrm{~s}^{-1}$ respectively $\left(\mathrm{K}_{\mathrm{m}}=15 \mu \mathrm{M}\right.$ for actin), compared with $0.11 \mathrm{~s}^{-1}$ and $0.9 \mathrm{~s}^{-1}$ respectively $\left(\mathrm{K}_{\mathrm{m}}\right.$ $=8 \mu \mathrm{M}$ for actin) for the unlabelled cysteine construct and $0.07 \mathrm{~s}^{-1}$ and $2.2 \mathrm{~s}^{-1}\left(\mathrm{~K}_{\mathrm{m}}=59\right.$ $\mu \mathrm{M})$ for a wild-type construct ${ }^{19}$. It appears that the mutagenesis has a dominant effect compared with labelling with pyrene per se, however the unlabelled double cysteine construct may be prone to oxidation under to assay conditions.

Fluorescence measurements. Steady-state fluorescence measurements were carried out using an SLM 48000 spectrofluorometer. Pyrene was excited at $365 \mathrm{~nm}$ with a $\mathrm{Hg}-\mathrm{Xe}$ lamp (Xe lamp was used for excitation spectra). Stopped-flow transients were recorded on an Applied Photophysics SX18MV instrument, using a $455 \mathrm{~nm}$ cut-off filter to record pyrene excimer emission. The apparatus was modified to allow acquisition of two emission signals (e.g. excimer and light scatter) simultaneously. In general, changes in fluorescence of $<10 \%$ were more reliably detected in the stopped-flow apparatus than by manual addition using the fluorometer. Unless otherwise stated, spectrophotometric assays were carried out as described previously ${ }^{19}$. Fluorescence lifetime measurements were recorded using an Applied Photophysics LKS60 laser spectrophotomer. Although 
this apparatus was designed for flash photolysis, the long lifetime of the pyrene excimer could be recorded directly from a single flash of the Nd-YAG laser at $355 \mathrm{~nm}$.

1. Lymn, R. W. \& Taylor, E. W. Mechanism of adenosine triphosphate hydrolysis by actomyosin. Biochemistry 10, 4617-4624 (1971).

2. Fisher, A. J. et al. X-ray structures of the myosin motor domain of Dictyostelium discoideum complexed with MgADP.BeFx and MgADP.AlF4. Biochemistry 34, 8960-8972 (1995).

3. Dominguez, R., Freyzon, Y., Trybus, K. M. \& Cohen, C. Crystal structure of a vertebrate smooth muscle myosin motor domain and its complex with the essential light chain: visualization of the pre- power stroke state. Cell 94, 559571 (1998).

4. Geeves, M. A. \& Holmes, K. C. Structural mechanism of muscle contraction. Anпu Rev Biochem 68, 687-728 (1999).

5. Volkmann, N. et al. Evidence for cleft closure in actomyosin upon ADP release. Na.t Struct. Biol. 7, 1147-1155 (2000).

6. Himmel, D. M. et al. Crystallographic findings on the internally uncoupled and near-rigor states of myosin: Further insights into the mechanics of the motor. Proc Natl Acad Sci U S A 24, 12645-12650 (2002).

7. Holmes, K. C., Angert, I., Kull, F. J., Jahn, W. \& Schroeder, R. R. Electron cryo-microscopy reveals how myosin strong binding to actin releases nucelotide. Nature in press (2003).

8. Reubold, T. F., Eschenburg, S., Becker, A., Kull, F. J. \& Manstein, D. J. A structural model for actin-induced nucleotide release in myosin. Nat. Struct. Biol. in press (2003).

9. Coureux, P. D. et al. The myosin V motor visualized at 2.0A without bound nucleotide reveals a new structural state of myosin. Nature in press (2003).

10. Rayment, I. et al. Three-dimensional structure of myosin subfragment-1: a molecular motor. Science 261, 50-58 (1993).

11. Houdusse, A., Szent-Gyorgyi, A. G. \& Cohen, C. Three conformational states of scallop myosin S1. Proc Natl Acad Sci U S A 97, 11238-11243 (2000). 
12. Rayment, I. et al. Structure of the actin-myosin complex and its implications for muscle contraction. Science 261, 58-65 (1993).

13. Shih, W. M., Gryczynski, Z., Lakowicz, J. R. \& Spudich, J. A. A FRET-based sensor reveals large ATP hydrolysis-induced conformational changes and three distinct states of the molecular motor myosin. Cell 102, 683-694 (2000).

14. Yengo, C. M., De La Cruz, E. M., Chrin, L. R., Gaffney, D. P., 2nd \& Berger, C. L. Actin-induced closure of the actin-binding cleft of smooth muscle myosin. $J$ Biol Chem 277, 24114-24119 (2002).

15. Wakelin, S. et al. Engineering Dictyostelium discoideum myosin II for the introduction of site-specific probes. J. Muscle Res .Cell Motil. 23, 673-683 (2002).

16. Birks, J. B., Kazzaz, A. A. \& King, T. A. 'Excimer' fluorescence IX. Lifetime studies of pyrene crystals. Proc. Roy. Soc. (London) A 291, 556-569 (1966).

17. Lehrer, S. S. Intramolecular pyrene excimer fluorescence: a probe of proximity and protein conformational change. Methods Enzymol 278, 286-295 (1997).

18. Kuhlman, P. A. \& Bagshaw, C. R. ATPase kinetics of the Dictyostelium discoideum myosin II motor domain. J. Muscle Res. Cell Motil. 19, 491-504 (1998).

19. Malnasi-Csizmadia, A., Woolley, R. J. \& Bagshaw, C. R. Resolution of conformational states of Dictyostelium myosin II motor domain using tryptophan (W501) mutants: Implications for the open-closed transition identified by crystallography. Biochemistry 39, 16135-16146 (2000).

20. Kovacs, M., Malnasi-Csizmadia, A., Woolley, R. J. \& Bagshaw, C. R. Analysis of Nucleotide Binding to Dictyostelium Myosin II Motor Domains Containing a Single Tryptophan Near the Active Site. J. Biol. Chem. 277, 28459-28467 (2002).

21. Geeves, M. A., Jeffries, T. E. \& Millar, N. C. ATP-induced dissociation of rabbit skeletal actomyosin subfragment 1 . Characterization of an isomerization of the ternary acto-S1-ATP complex. Biochemistry 25, 8454-8458 (1986).

22. Taylor, E. W. Kinetic studies on the association and dissociation of myosin subfragment 1 and actin. J Biol Chem 266, 294-302 (1991). 
23. Cremo, C. R. \& Geeves, M. A. Interaction of actin and ADP with the head domain of smooth muscle myosin: implications for strain-dependent ADP release in smooth muscle. Biochemistry 37, 1969-1978 (1998).

24. Geeves, M. A. Dynamic interaction between actin and myosin subfragment 1 in the presence of ADP. Biochemistry 28, 5864-5871 (1989).

25. Manstein, D. J., Schuster, H. P., Morandini, P. \& Hunt, D. M. Cloning vectors for the production of proteins in Dictyostelium discoideum. Gene 162, 129-134. (1995).

26. Manstein, D. J. \& Hunt, D. M. Overexpression of myosin motor domains in Dictyostelium: screening of trans formants and purification of the affinity tagged protein. J Muscle Res Cell Motil 16, 325-232 (1995).

27. Malnasi-Csizmadia, A. et al. Kinetic resolution of a conformational transition and the ATP hydrolysis step using relaxation methods with a Dictyostelium myosin II mutant containing a single tryptophan residue. Biochemistry 40, 12727-12737 (2001).

28. Sarker, G. \& Sommer, S. S. The "megaprimer" method of site-directed mutagenesis. Biotechniques 8 (1990).

<received >

Acknowledgements. We thank William Shih and Jim Spudich for the cysteinedeficient construct and Ken Holmes and Rasmus Schroeder for the coordinates of their actomyosin model. We are grateful to the Wellcome Trust, BBSRC, National Science Foundation and the Magyary Zoltán Foundation for financial support.

Competing interests statement The authors declare that they have no competing financial interest.

Correspondence and address for materials should be addressed to CRB (email: $\underline{\text { crb5@le.ac.uk) }}$

Figure 1. Models to show the location of Dd residues 416 (cyan) and 537 (yellow) that are labelled with pyrene. Skeletal muscle myosin structures are shown in (a) and (b) to 
facilitate comparison with the predicted cleft-closed structure of Holmes et $\mathrm{al}^{7}$. (a) apo skeletal myosin $(2 \mathrm{MYS})^{10}$. (b) rigor structure ${ }^{7}$ showing that residues equivalent to 416 and 537 move closer (c) Part of the upper and lower Dd $50 \mathrm{~K}$ domain structure with an antiparallel pyrene dimer in the open cleft state $(1 \mathrm{MMD})^{2}$. (d) Equivalent region in the modeled closed-cleft structure ${ }^{7}$ with a parallel pyrene dimer. The pyrene positions were determined from molecular dynamics simulations using Discover3 (Insight, MSI). Color code: $\mathrm{N}$-terminal $25 \mathrm{~K}$ domain, green; upper $50 \mathrm{~K}$ domain, red; lower $50 \mathrm{~K}$ domain white; C-terminal $20 \mathrm{~K}$ domain, blue; Actin subunits, orange, dark green and magenta.

Figure 2. Fluorescence emission spectra of pyr-Md (365 nm excitation). (a) $0.4 \mu \mathrm{M}$ pyr-Md alone (solid line), in the presence of $2 \mu \mathrm{M}$ F-actin (dashed line) and after addition of $1 \mathrm{mM} \mathrm{MgATP}$ to the acto-pyr-Md complex (dotted line). (b) $0.4 \mu \mathrm{M}$ pyr-Md of the apoenzyme (solid line) and in the presence of $1 \mathrm{mM} \mathrm{MgATP}$ in the absence of actin (dotted line). Denaturation with $1 \mathrm{M}$ guanadinium hydrochloride (dashed line). Buffer conditions: $40 \mathrm{mM} \mathrm{NaCl}, 20 \mathrm{mM}$ Hepes, $2 \mathrm{mM} \mathrm{MgCb}, \mathrm{pH} 7.5,20{ }^{\circ} \mathrm{C}$.

Figure 3. Time-resolved pyrene fluorescence measurements. (a) Excimer fluorescence lifetimes measured using a single successive laser pulses at $355 \mathrm{~nm}$ with $1 \mu \mathrm{M}$ pyrMd (solid line), after addition of $2 \mu \mathrm{M}$ actin (dashed line), followed by $1 \mathrm{mM}$ ATP (bold dotted line). The monochromator was set at $480 \mathrm{~nm}$ to detect excimer fluorescence emission. The lamp pulse (dotted line) was determined using scattered light (monochromator set to $355 \mathrm{~nm}$ ) and was normalized to the pyr-Md fluorescence trace to show the near coincident rise time (b) time-resolved pyrene emission spectra for pyr-Md constructed by repeat excitation pulses $(355 \mathrm{~nm})$ with the emission monochromator set between 370 and $520 \mathrm{~nm}$ at $5 \mathrm{~nm}$ intervals. The records are corrected for the background signal from buffer alone and photobleaching of $2 \%$ per pulse. Successive traces were constructed at $20 \mathrm{~ns}$ intervals starting $10 \mathrm{~ns}$ from the beginning of the lamp pulse (except the final bottom trace which is at $180 \mathrm{~ns}$ ). Note that the decay of the monomer peaks is significantly faster than the excimer peak and is limited by the lamp pulse width. 
Figure 4. Stopped-flow records of pyrene excimer fluorescence on interaction of pyrMd with actin and nucelotides. (a) Fluorescence (black solid line) and light scatter (blue dashed line) on mixing $0.5 \mu \mathrm{M}$ pyr-Md with $5 \mathrm{uM}$ actin in the absence of nucleotide. Fitted exponential $=1.6 \mathrm{~s}^{-1}$ (blue solid line). Premixing the pyr-Md with $10 \mu \mathrm{M}$ ADP slowed down the reaction by less than 2-fold. (b) Fluorescence (solid line) on mixing $500 \mu \mathrm{M}$ ATP with $1 \mu \mathrm{M}$ pyr-Md in the absence (red trace) and presence (black trace) of $2 \mu \mathrm{M}$ actin. Light scatter (blue dashed line) recorded simultaneously with fluorescence. (c) Normalized fluorescence (solid lines) and inverted light scatter (dashed lines) on mixing $1 \mu \mathrm{M}$ pyr-Md $+2 \mu \mathrm{M}$ actin with $500 \mu \mathrm{M}$ ATP (black), $500 \mu \mathrm{M}$ ATP $\gamma \mathrm{S}$ (blue) or $1 \mathrm{mM}$ ADP (red). The amplitude of the changes with ATP $\gamma \mathrm{S}$ and ADP were $95 \%$ and $55 \%$ of those with ATP, respectively. All concentrations refer to the reaction chamber. 

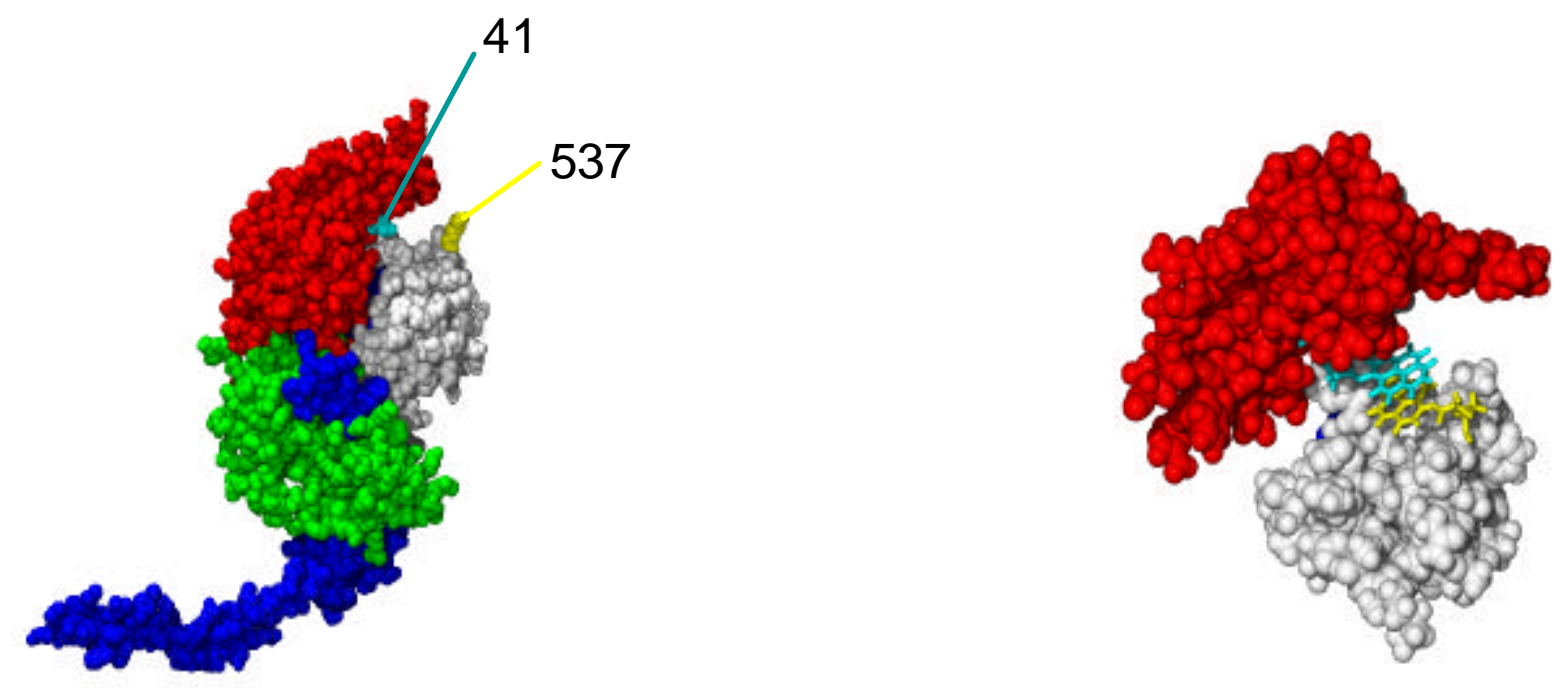

537
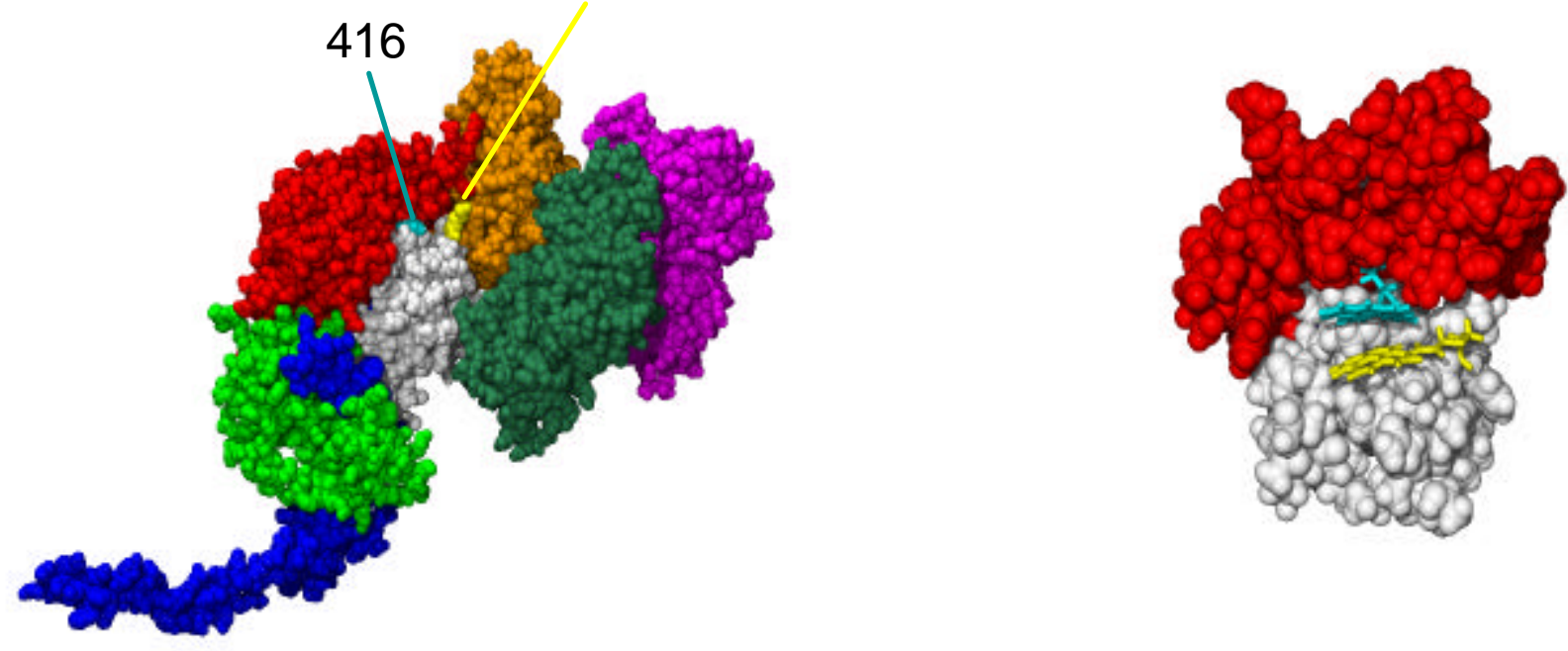

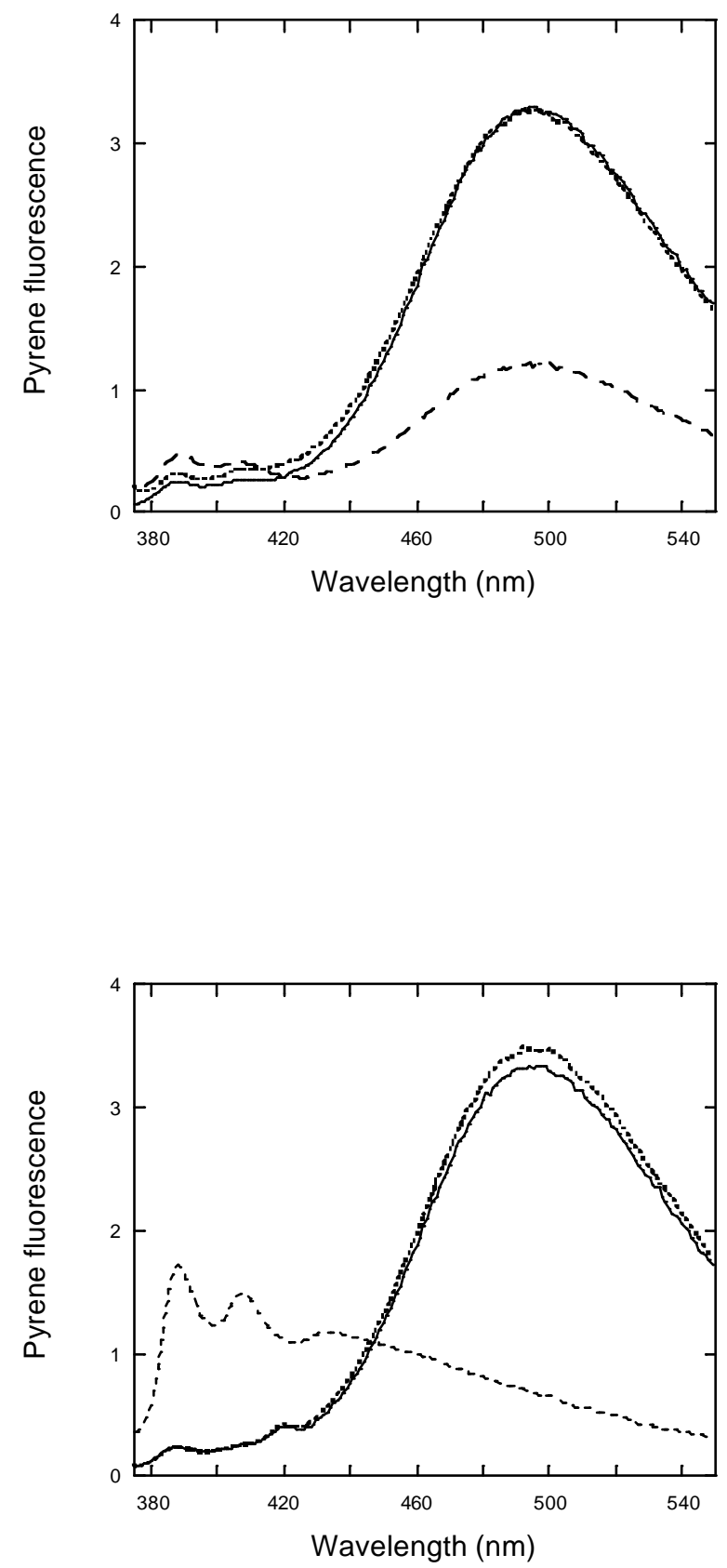
16
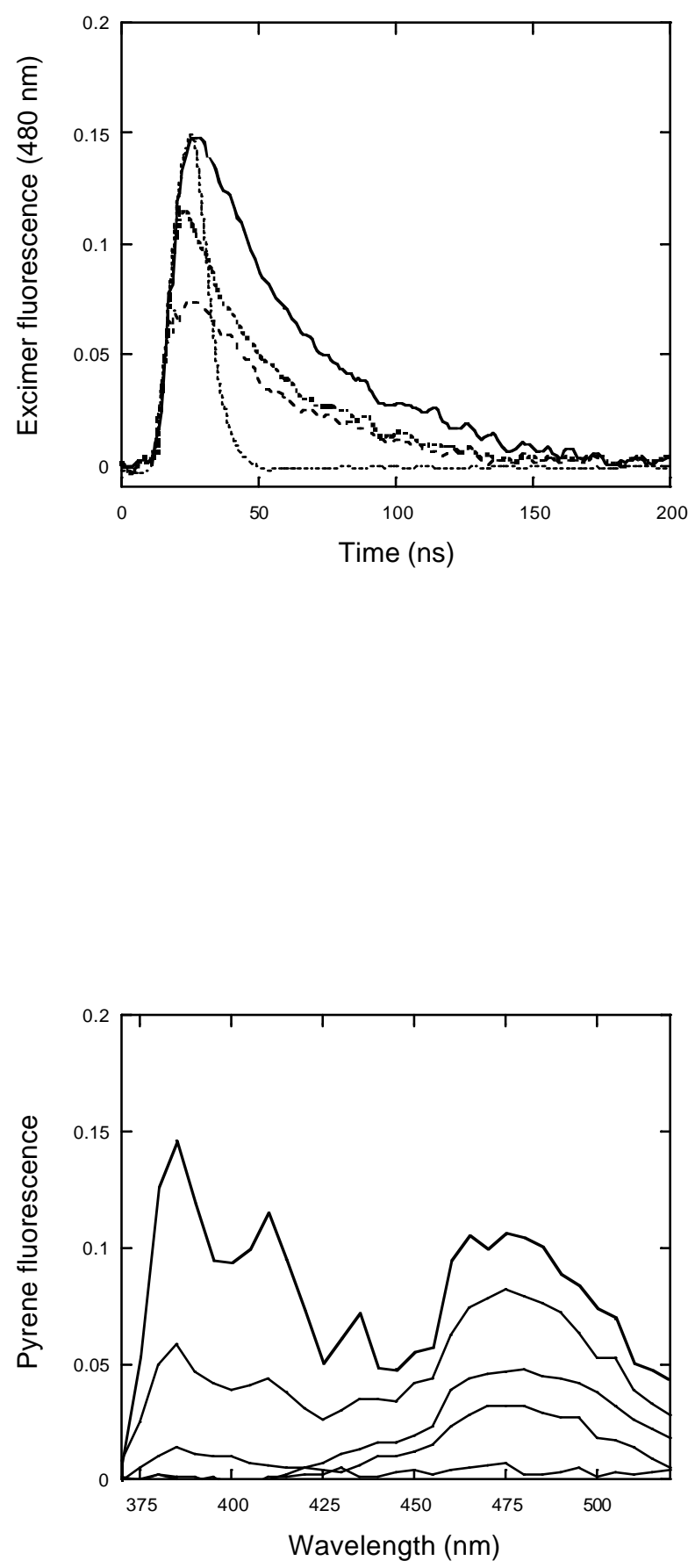

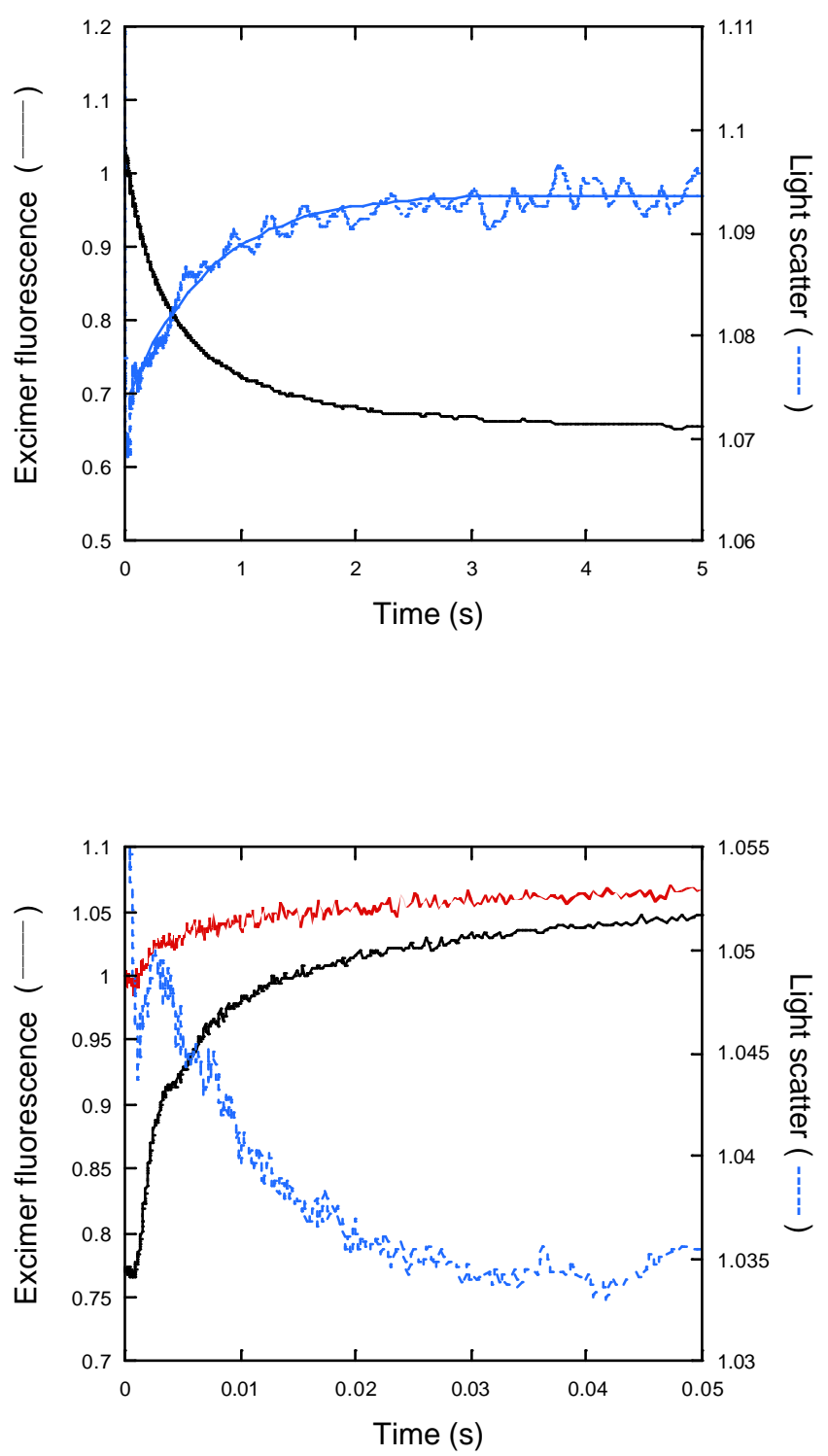


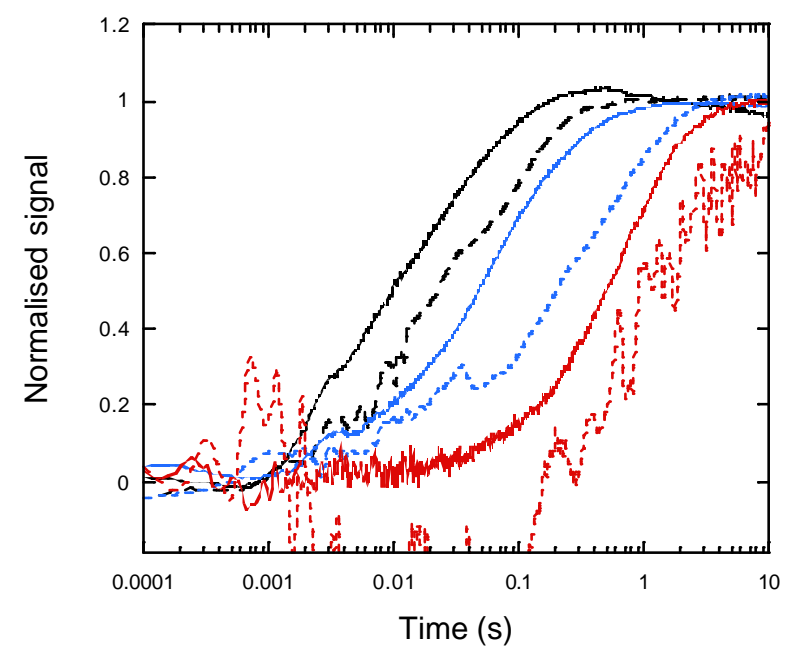

